\title{
Optical Many Casting Using QoS Depend Layer Aware Mechanism
}

\author{
R.C. Arun Chander \\ PG scholar, Dept of Electronics \& communication, K.L.N college of Engineering, Madurai, India \\ arun_chan@yahoo.com \\ P.Karunakaran \\ Assistant Professor, Department of ECE, K.L.N College of Information Technology, Madurai, India \\ karunakaranvkp@gmail.com \\ S.Venkatraman \\ Assistant Professor, Department of ECE, Vel Tech Chennai, India \\ venkiv118@gmail.com \\ I.Hameem Shanavas \\ Assistant Professor, Department of ECE, M.V.J College of Engineering, Bangalore-67, India \\ hameemshan@gmail.com
}

\begin{abstract}
Many distributed applications require a group of destinations to be coordinated to a single source. Multicasting is a communication paradigm to implement these distributed applications. In multicasting, at least one of the member in the group cannot satisfy the service requirement of the application, the multicast request said to be blocked. On the contrary in manycasting, destinations can join or leave the group, depending upon whether it satisfies the service requirement or not. Manycasting is performed over optical burst-switched (OBS) networks based on multiple qualities of service (QoS) constraints. The multiple constraints can be in the form of physical layer impairments, transmission delay, and reliability of the link. Destinations qualify only if they satisfy the Qos constraints. We develop a simple yet efficient routing algorithm which is based on the classic shortest path algorithm. The proposed layer aware FEC (L-FEC) generates repair symbols so that protection of less important dependency layers can be used with protection of more important layers for combined error correction.
\end{abstract}

Index terms - BER, Constraint Based Routing (CBR), Manycast, Optical Burst-Switched Networks (OBS), Quality Of Service, Qos Routing, WDM

\section{Introduction}

Manycast is also called Quorumcast And Was First Proposed. Many casting is a generalization of multicasting, in which the group of destinations that receive the message are to be selected instead of being given. In manycasting messages are sent to a subset of destinations (quorum pool), which are selected from set (quorum group), such that. A manycast request is said to be successful if any of them participate in that session. A quorum pool is a majority group and hence we always require. Manycasting is also a generalization of anycasting where the message needs to be delivered to any one of the group. However, in this case and the above inequality will not be valid for anycasting. Manycasting has caught the attention of several researchers during the recent past, due to the emergence of many distributed applications .Distributed applications, such as video conferencing, distributed interactive simulations (DIS), grid computing, storage area network (SAN), and distributed content distribution network $(\mathrm{CDN})$ require large amounts of bandwidths and an effective communication between single source and a set of destinations. Manycasting is also an attractive and viable communication paradigm for providing fault tolerance for the defense information infrastructures in the battlefield. Provisioning of connections based on QoS to these applications is an important issue.

QoS can include delays incurred during transmission, reliability, and signal degradation. For example reliability is an important issue in designing SANs. Since SANs are supported over fiber-channel (FC), threat to failure can occur due to cable cuts, physical attacks, and catastrophic effects. Grid applications depends on the QoS that a network can provide to ensure successful completion of the job . To meet the demands of such distributed applications there is an emergence of intelligent optical control plane architectures. WDM networks include optical circuit switching (OCS), optical packet switching (OPS), and optical burst switching (OBS). In OCS a light path is 
set up by the user for the entire duration of the data transfer. In OPS the user data is transmitted in optical packets that are switched entirely in optical domain. In OBS the user data is transmitted all-optically as bursts with the help of an electronic control plane. One of the primary issues with OCS is that the link bandwidth is not utilized efficiently in the presence of Bursty traffic. On the other hand, many technological limitations have to be overcome for OPS to be commercially viable.

OBS networks overcome the technological constraints imposed by OPS and the bandwidth inefficiency of OCS networks. In this paper, we focus on the optical transport network being OBS. The proposed algorithms can easily be modified to work for OCS and OPS networks. OBS networks are well suited for supporting delay-sensitive computationally intensive grid applications known as Grid OBS (GOBS).

Apart from supporting manycasting over optical networks, we also need to provision QoS in OBS networks. This is because QoS provisioning methods in IP will not apply to the optical counterpart, as there is no store-and-forward model Such mechanisms for QoS provisioning in IP over OBS networks must consider the physical characteristics and limitations of the optical domain. Physical characteristics of the optical domain include optical-signal degradation, propagation delay incurred from source to destination, and link reliability from $q$ catastrophic effects. As the optical signal traverses in the transparent optical network, with the absence of electrical regenerators there will be significant loss of power due to much impairment.

These impairments can be attenuation loss, multiplexer/demultiplexer loss, optical-cross-connect switch loss (OXC), and split loss (for multicast capable switches).ASE noise present in the EDFAs decreases the optical-signal-to-noise ratio (OSNR). Decrease in OSNR increases bit error rate (BER) of the signal. Hence, the signal is said to lost if BER is more than the required threshold. $3 R$ regeneration of optical signal resets the effects of nonlinearity, fiber dispersion, and amplifier noise, without introducing any additional noise. This $3 \mathrm{R}$ regeneration requires retiming and clock recovery system, which cannot easily be carried alloptically. Hence, O/E/O conversion becomes inevitable. Delay accumulation due to $\mathrm{O} / \mathrm{E} / \mathrm{O}$ conversions can be significant when compared to the propagation delay in OBS networks. Wavelength regeneration can also result in reliability reduction and operational cost increase The MCM-DM is given in Algorithm 2. It contains two procedures (1) for calculating the QoS parameters and updating BHP with the new values, defined as Procedure. QoS(1) and (2) for calculating the number of destinations that can be reached from the next-hop node is greater than, defined as Procedure. Block. Instead of discarding destinations as in MCM-SPT,

We keep these destinations as secondary destinations and use them if any of the first are blocked. Intuitively, one can understand that request blocking could be reduced in the case of MCM-DM as members in the quorum pool are added or removed dynamically. While adding the destinations into the quorum pool the burst traversal can be along a longer path, deteriorating certain QoS parameters. The resulting QoS blocking could be high when compared to MCM-SPT.

\section{Service Attributes}

We define $\gamma_{j}, \eta_{j}$ and $\tau_{\mathrm{j}}$ as noise factor, reliability factor and end-to-end propagation delay for the $\operatorname{Link} j$, respectively. Noise factor is defined as ratio of input OSNR and the output signal to noise ratio, thus we have

$$
\eta=\frac{\operatorname{OSNR}_{i / p}}{\operatorname{OSNR}_{o / p}}
$$

Where is defined as the ratio of the average signal power received at a node to the average ASE noise power at that node. The OSNR of the link and factor are related as

$$
\mathrm{q}=\frac{2 \sqrt{\frac{B_{o}}{B_{e}}} O S N R}{1+\sqrt{1+4 O S N R}}
$$

Where $B_{o}$ and $B_{e}$ are optical and electrical bandwidths, respectively. Bit-error rate is related to the - factor as follows

$$
\operatorname{BER}=\frac{1}{2} \operatorname{erfc}\left(\frac{q}{\sqrt{2}}\right)
$$

The overall noise factor of a burst that has traversed $H$ hops is given by

$$
\eta_{H}=\prod_{k=1}^{H} \eta_{k}
$$

The end-to-end reliability for the path traversing $H$ hops is calculated as

$$
\gamma_{H}=\prod_{k=1}^{H} \gamma_{\mathrm{K}}
$$

If is the propagation delay of a link $\tau$, then end-toend Delay for hops, is given by

$$
\tau_{H}=\sum_{k=1}^{H} \tau_{K}
$$

\section{Path Information vector}

The service attributes can be used to maintain the local net- work information and by properly comparing these vectors, the destinations can be chosen. Comparison of multidimensional metrics can be done 
using the notion of lattices [25]. Lattices are explained using the ordering denoted by $\preccurlyeq$, which has the properties of reflexivity, anti-symmetry, and transitivity. We denote the information vector at link $j$ as,

$$
\Omega_{j}=\left(\begin{array}{c}
\eta_{j} \\
\lambda_{j} \\
\tau_{j}
\end{array}\right)
$$

\section{Architecture Model}

$(800,0.9)$

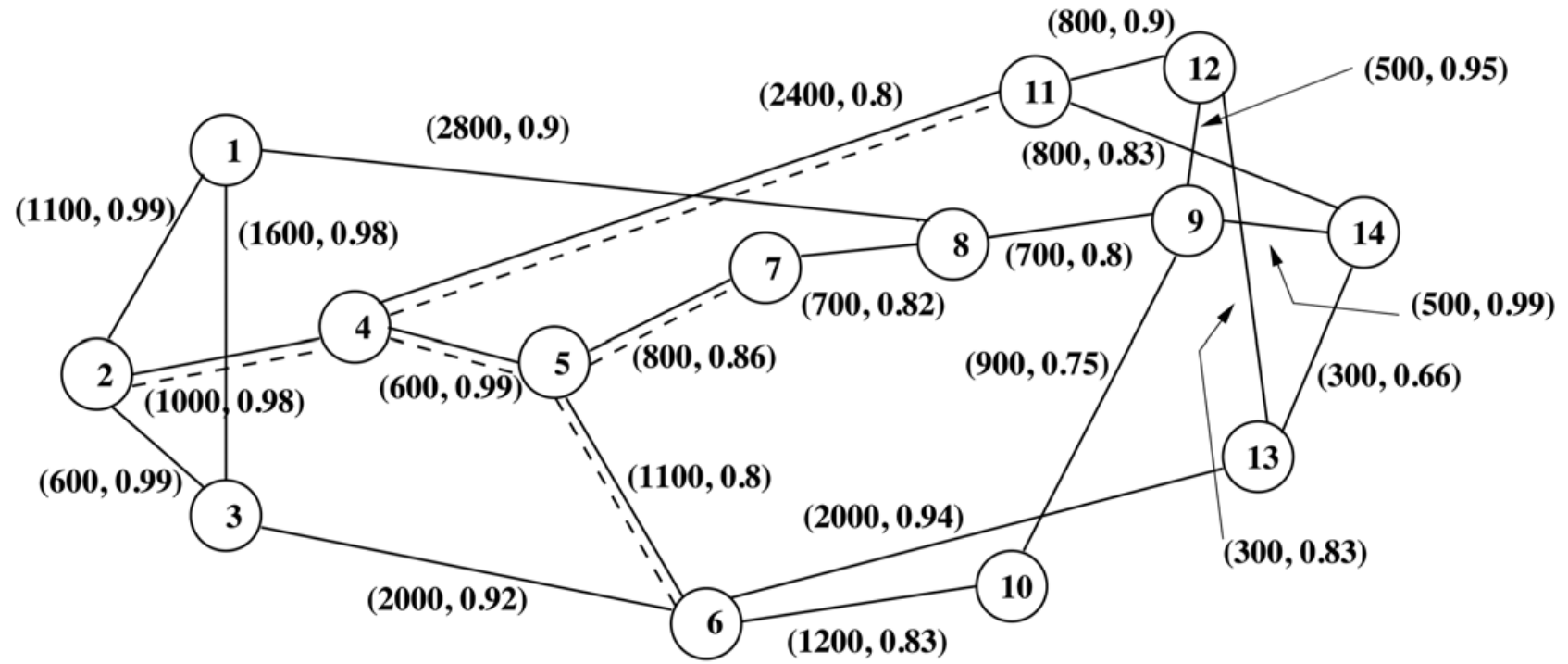

Fig.1: NSF network with 14 nodes and 21 bidirectional links. The weights represent distance in km and the corresponding reliability

\section{Algorithm 1 Multi-Constraint Manycast-Shortest Path Tree (MCM-SPT)}

Input: The manycast request $\left(i d, u, D_{u}, \kappa_{u}, \mathrm{~T}^{\left(\theta_{p}\right)}, \Omega_{\langle u-1, u\rangle}\right)$ arrives at the source node $u$ with a candidate destination set $D_{u}$, along with the $\kappa$ intended.

Output: Manycast request to the next-hop (or child) node after satisfying QoS parameters for the service $\theta_{p}$.

I: Initialization: When the burst first enters the network with the request $\left(s, D_{s}, \kappa_{s}, \top^{\left(\theta_{p}\right)}\right)$, we tag the request with a burst ID and $\Omega_{\text {initial }}$, where $\Omega_{\text {initial }}=[1,1,0]^{T}$. We therefore have the request as $\left(i d, s, D_{s}, \kappa_{s}, \top^{\left(\theta_{p}\right)}, \Omega_{\text {initial }}\right)$.

2: if $u \in D_{u}$ then

3: $D_{u} \leftarrow D_{u} \backslash\{u\}$;

4: $\quad \kappa_{u} \leftarrow \kappa_{u}-1$;

5: else

6: $\quad D_{u}^{\prime} \leftarrow$ SORT.SP $\left[D_{u}\right]$;

7: for $j \leftarrow 1$ to $\kappa_{u}$ do

8: $\quad n_{j} \leftarrow N E X T . H O P . N O D E . S P\left[u, d_{j}^{\prime}\right]$;

9: $\quad N=N \cup\left\{n_{j}\right\}$;

10: end for

11: for $j \leftarrow 1$ to $|N|$ do

12: $\quad$ if $L I N K\left\langle u, n_{j}\right\rangle=F R E E$ then

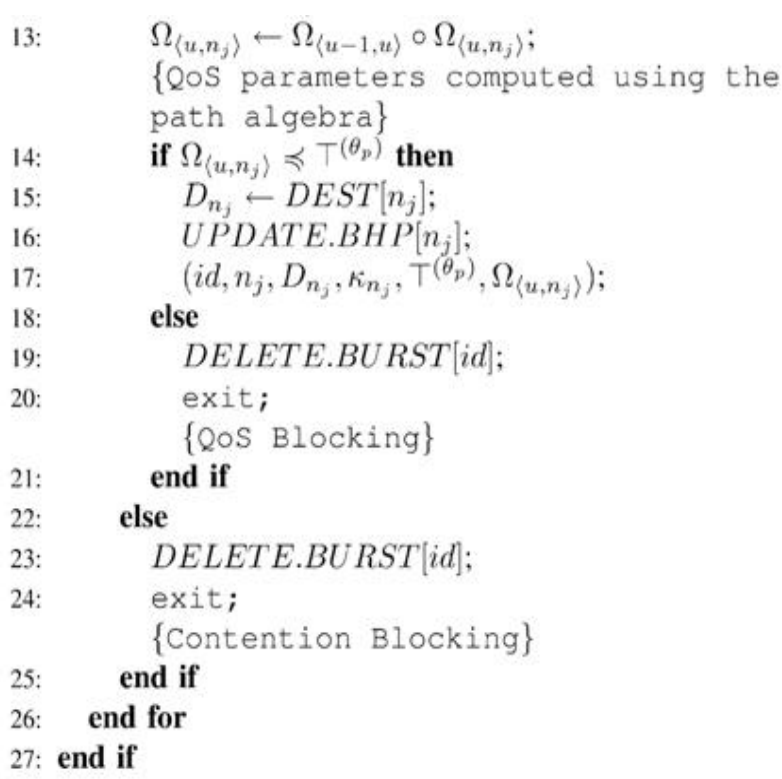

\section{Proposed System}

We propose a simple yet efficient routing algorithm for manycasting, which is based on the classic shortestpath tree algorithm. Our discussion shows that, by using multicast capable OXCs, only minor changes are needed for the well-studied OBS network architecture which aims at unicasting to support manycasting service. In this project, this work proposes a method for extending forward error correction (FECs) codes 
following dependency structures within the media. The proposed layer-aware FEC (L-FEC) generates repair symbols so that protection of less important dependency layers can be used with protection of more important layers for combined error correction. We propose algorithms that provide QoS-based manycasting over OBS networks. We also develop a mathematical problem formulation for manycast destination selection policies based on QoS constraints as required by certain applications. Our approach can incorporate multiple constraints related to different services. The proposed methods are service- centric and completely decentralized, as they use only localnetwork state information. Performance analysis of end-to-end propagation delay and blocking probability for OBS based grids using anycasting has been presented. Different types of anycasting algorithms has been compared in with the shortest-path unicast routing, where the destinations has a specific address. Manycasting over OBS networks based on multiple resources have been addressed.

\section{Optical Burst-Switched Networks}

The many cast problem, also referred to as the quorum cast problem and the k-Steiner tree problem, was first proposed in 1994 independently and is defined as follows: given a network $\mathrm{G}(\mathrm{V} ; \mathrm{E})$, an edge cost function $\mathrm{g}: \mathrm{E} ! \mathrm{R}+$, an integer $\mathrm{k}$, a source $\mathrm{s}$, and a subset of candidate destinations Dc $\mu \mathrm{V}, \mathrm{jDcj}=\mathrm{m}, \mathrm{k}$, find a minimum cost tree spanning $\mathrm{k}$ destinations in Dc. The cost of a tree is defined as a sum of the cost of edges on the tree. A manycast request can simply be denoted as $(\mathrm{s} ; \mathrm{Dc} ; \mathrm{k})$. A subtle difference between manycast and multicast is that, in manycast, the actual destinations to be covered are to be determined instead of being given as in multicast. The manycast problem is NP-hard [2]. To support manycasting service in IP over optical burst switched (OBS) networks, we first need to decide which layer should be responsible for selecting $\mathrm{k}$ out of $\mathrm{m}$ candidate destinations. If the selection is done at the IP layer, manycast requests become multicast requests to the OBS layer, and it is sufficient that OBS networks support only multicasting service. However, for an overlay network architecture, which is the most used network architecture in practice, the IP layer usually does not have much information about the OBS layer. Then the selection of $\mathrm{k}$ destinations by the IP layer is similar to the random algorithm, which has been proved to have poor performance. Therefore, supporting manycasting at the OBS layer is necessary for bandwidth-efficient manycasting.

\section{Qos (Quality Of Service)}

In the field of computer networking and other packet-switched telecommunication networks, the traffic engineering term quality of service (QoS) refers to resource reservation control mechanisms rather than the achieved service quality. Quality of service is the ability to provide different priority to different applications, users, or data flows, or to guarantee a certain level of performance to a data flow. For example, a required bit rate, delay, jitter, packet dropping probability and/or bit error rate may be guaranteed. Quality of service guarantees are important if the network capacity is insufficient, especially for real-time streaming multimedia applications such as voice over IP, online games and IP-TV, since these often require fixed bit rate and are delay sensitive, and in networks where the capacity is a limited resource, for example in cellular data communication. QoS is sometimes used as a quality measure, with many alternative definitions, rather than referring to the ability to reserve resources. Quality of service sometimes refers to the level of quality of service, i.e. the guaranteed service quality. High QoS is often confused with a high level of performance or achieved service quality, for example high bit rate, low latency and low bit error probability.

\section{Result and Discussion}

Thus we obtain the result of blocking probability of both MCM shortest-path algorithms as well as for MCM-dynamic membership algorithm as follows. Our simulation results show that MCM-shortest path tree (MCM-SPT) algorithm performs better than MCMdynamic membership (MCM-DM) for delay constrained services and real time service, where as data services can be better provisioned using MCMDM algorithm .Results are shown below.

\section{Dataflow Diagram}

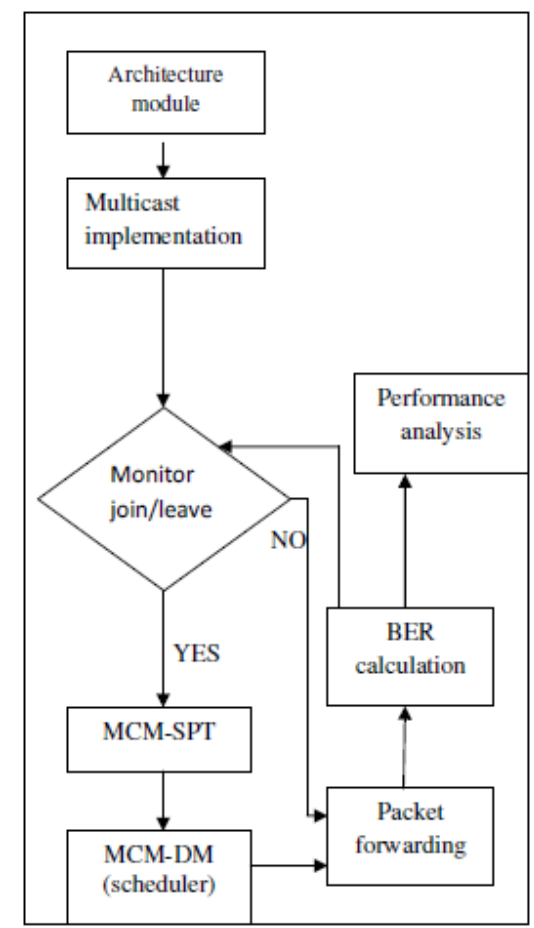


Time vs Packet loss

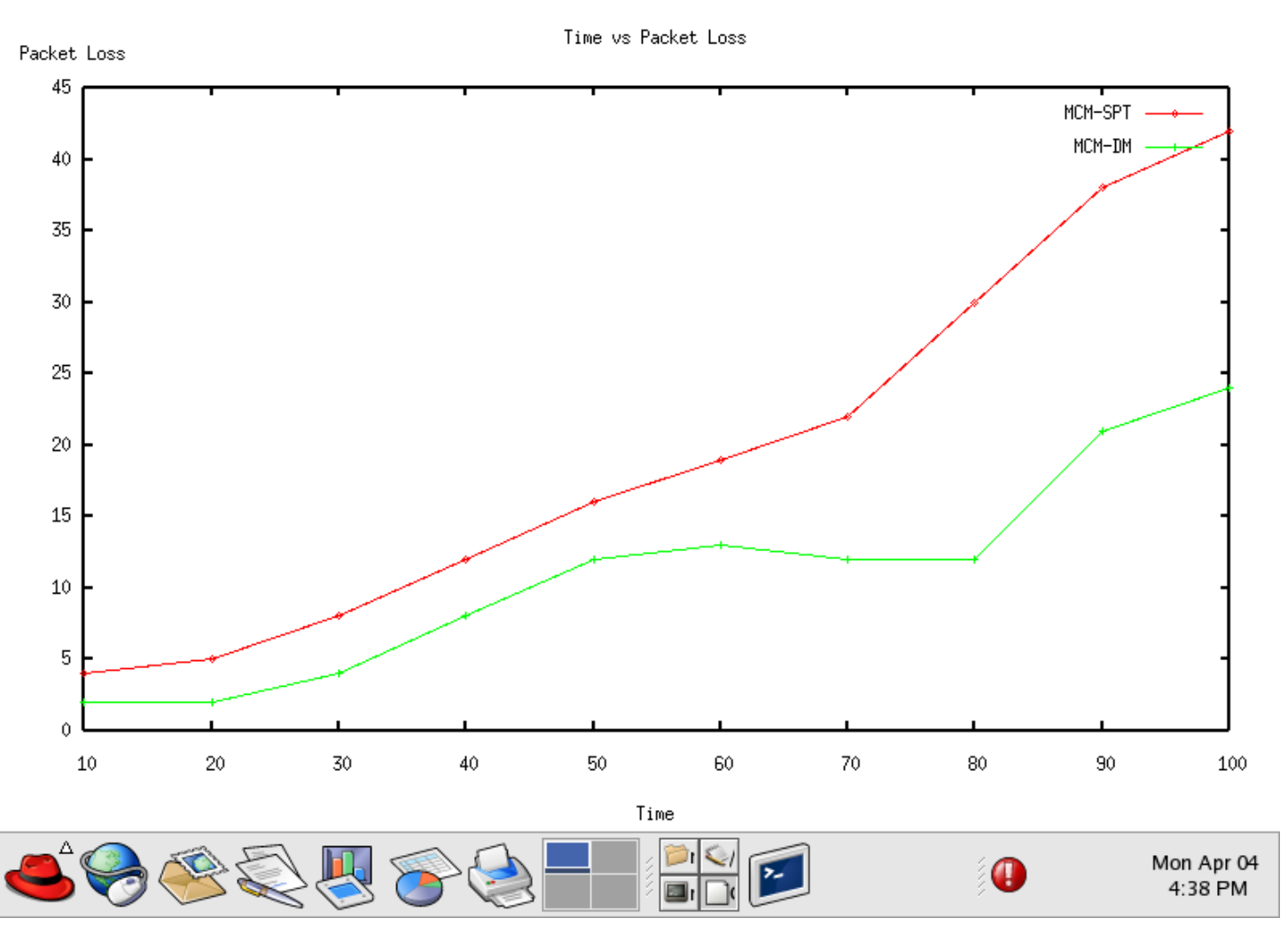

Time vs Throughput

\section{Gnuplot}

Time vs Throughput

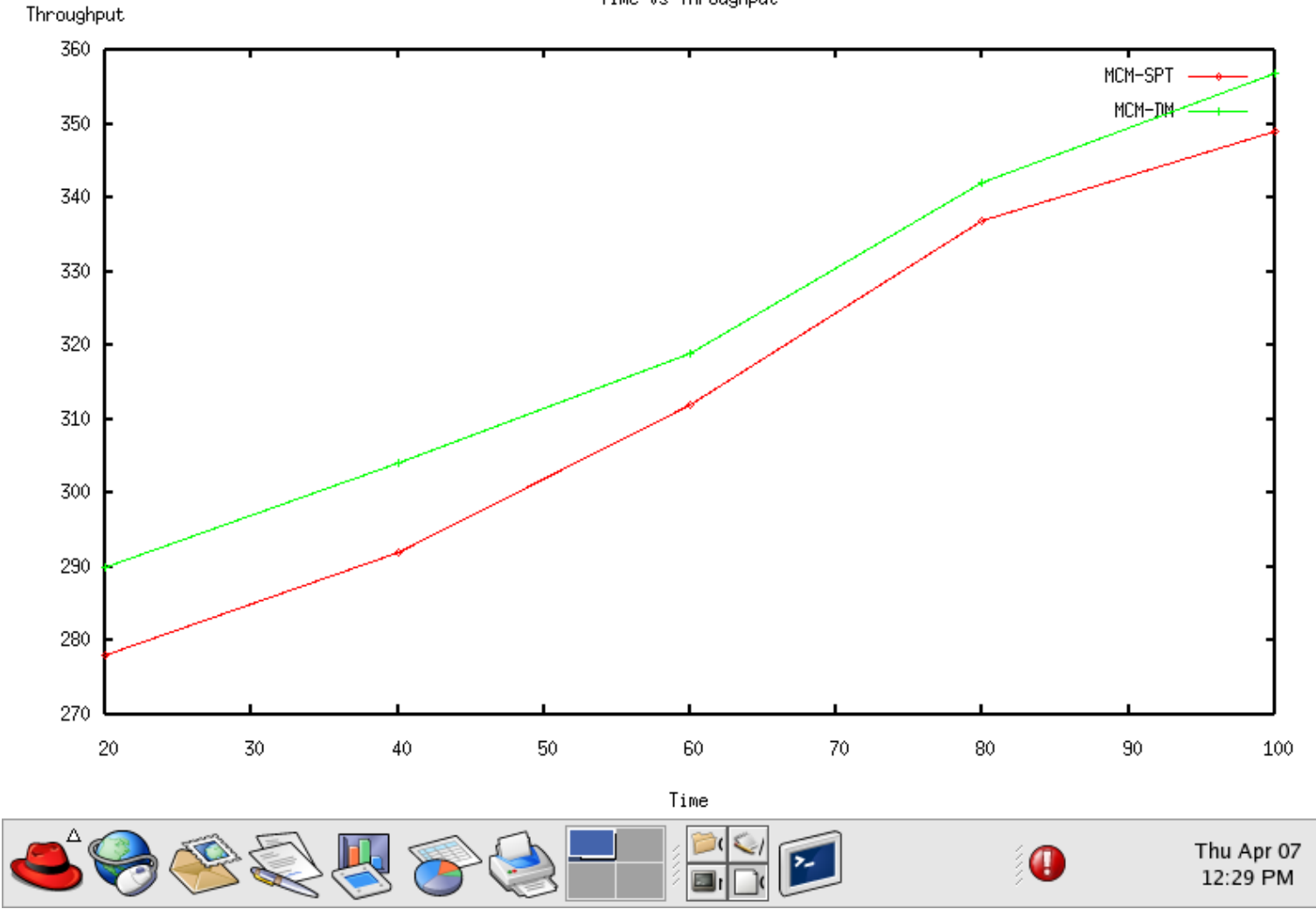


Load vs Delay

\section{Gnuplot}

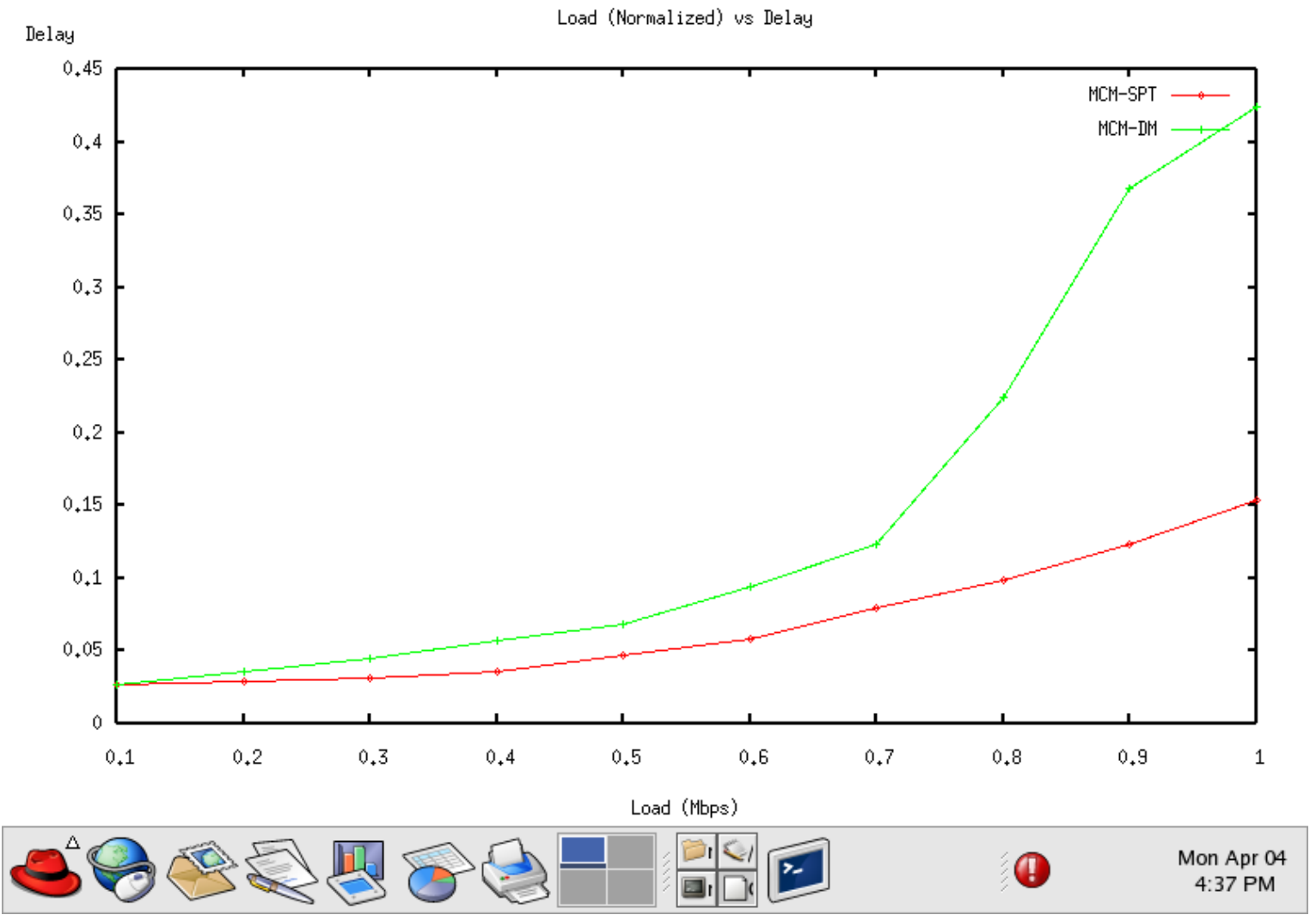

Load vs Request Blocking
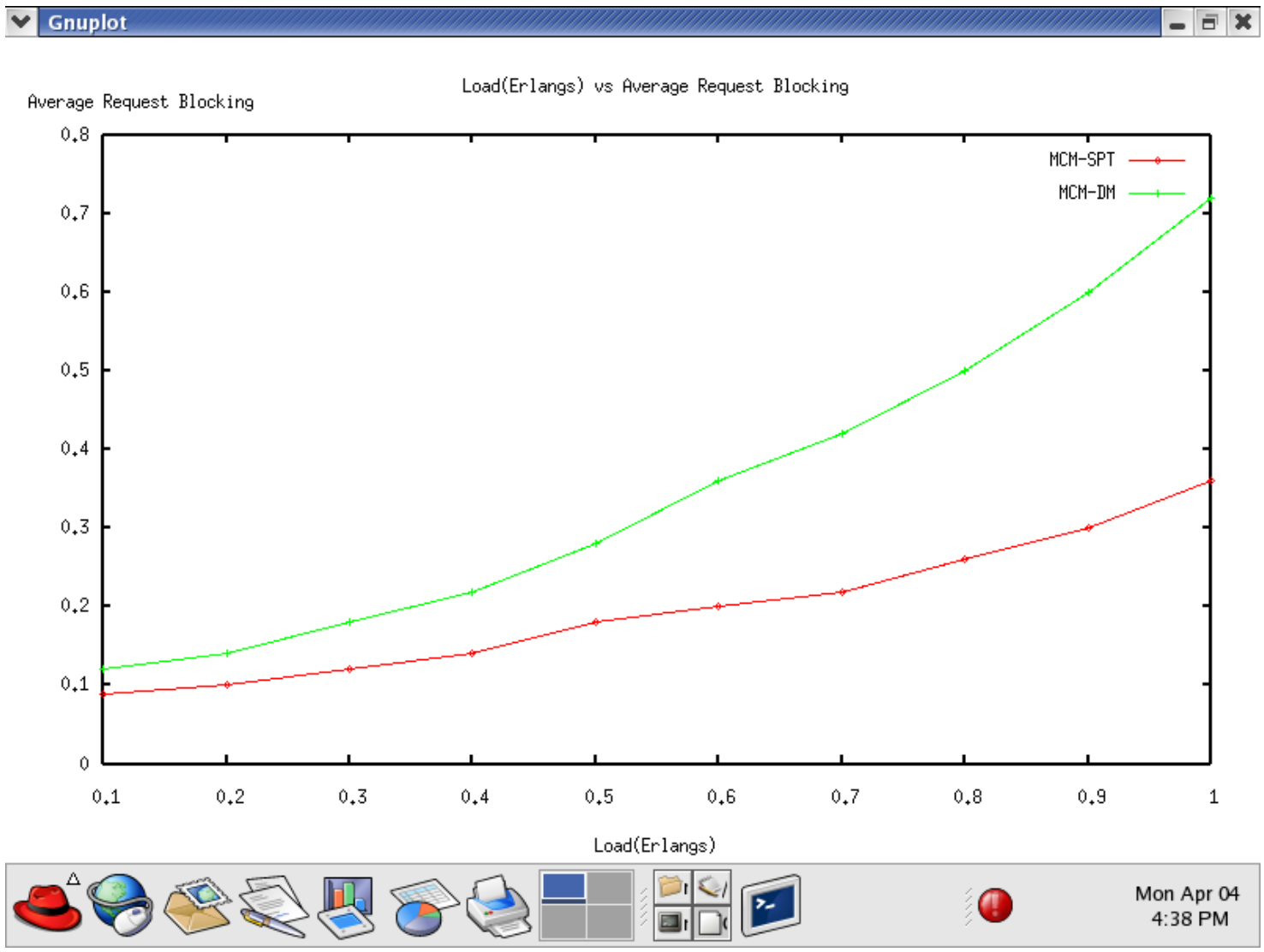


\section{Conclusion}

We discuss issues of impairment aware manycasting service over OBS networks. By incorporating q-field in the signaling we have implemented an easy way of updating the $\mathrm{q}$ depending on the signaling split at each node. This makes the algorithm work in the practical scenario where optical signal degrades due to physical layer impairments. We have accounted for the burst loss in both network layer and physical layer utilizing dynamically. We propose algorithms to support qosbased manycasting over optical burst-switched networks. Our qos model supports certain service parameters for the transmission of optical bursts, such as physical impairments, reliability, and propagation delay. We have developed a mathematical model based on lattice algebra for the multiconstraint manycast problem. By using distributed scheduling algorithms, bursts are routed to the destinations based on the contentions and qos conditions. We observe from the simulation results that multiconstrained manycast dynamic membership algorithm is suited for data service and multiconstrained manycast shortest path tree algorithm for real-time service. We also evaluated the performance of our algorithms for different manycast configurations. Our proposed manycasting algorithms can be easily adapted to facilitate other application service requirements. Our work can be further extended by considering sparse wavelength regenerations.

\section{References}

[1] Balagangdhar G.Bathula and vinod M.Vokkarane,"QOS-Based many casting over optical Burst switched (OBS) networks,", IEEE/ACM Transaction on Networking, FEB. 2010, pp. 271-283.

[2] N. Charbonneau, V. M. Vokkarane, R. Balasubramanian, and D. Silvia, "Mascot: Manycast architecture for service-oriented tactical applications," in Proc. IEEE Int. Conf. Technol. Homeland Secur., Waltham, MA, May 2009, pp. 171-176.

[3] B. G. Bathula, "QoS aware quorum casting over optical burst switched networks," Ph.D. dissertation, Indian Inst. Sci., Bangalore, India, 2008.

[4] X. Huang, Q. She, V. M. Vokkarane, and J. P. Jue, "Many casting over optical burst-switched (OBS) networks," in Proc. IEEE ICC, Glasgow, Scotland, May 2007, pp. 2353-2358.

[5] A. Jukan, "Optical control plane for the grid community," IEEE Commun. Surv. Tutor., vol. 9, no. 3, pp. 30-44, 2007.

[6] M. D. Leenheer, F. Farahmand, K. Lu, T. Zhang, P. Thysebaert, B. Volckaert, F. D. Turck, B. Dhoedt, P. Demeester, and J. P. Jue, "Anycast algorithms supporting optical burst switched grid networks," in Proc. IEEE ICNS, Silicon Valley, CA, Jul. 2006, pp. 63-69.

[7] X. Qiu, R. Telikepalli, T. Drwiega, and J. Yan, "Reliability and availability assessment of storage area network extension solutions," IEEE Commun. Mag., vol. 43, no. 3, pp. 80-85, Mar. 2005.

[8] A. Jukan and G. Franzl, "Path selection methods with multiple constraints in service-guaranteed WDM networks," IEEE/ACM Trans.Netw. vol. 12, no. 1, pp. 59-72, Feb. 2004.

[9] A. Jukan and G. Franzl, "Constraint-based path selection methods for on-demand provisioning in WDM networks," in Proc. IEEE INFOCOM, New York, Jun. 2002, pp. 827-836.

[10] L.Xu, H. G. Perros, and G. Rouskas, “Techniques for optical packet switching and optical burst switching," IEEE Commun. Mag., vol. 39, no. 1, pp. 136-142, Jan. 2001.

[11] S.Y.Cheung and A. Kumar, "Efficient quorumcast routing algorithms," in Proc. IEEE INFOCOM, Toronto, ON, Canada, Jun. 1994,pp. 840-847.

R.C.Arun Chander is Post Graduate Research Scholar in the Dept of Electronics \& communication, K.L.N college of Engineering, Madurai, India. His Research works includes Multicore processors, Multicasting, etc. Email:arun_chan@yahoo.com

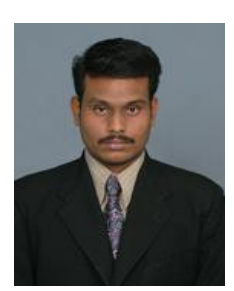

Karunakaran.P is currently working as Assistant Professor in the Department of ECE, K.L.N College of Information Technology, Madurai India. He has completed his Bachelor Degree in Electronics and Communication (2006), Masters in VLSI Design (2010). He had published few journals and attended many Conferences in National and International Level. His research areas are VLSI Testing, low Power Testing and Image processing. Email: karunakaranvkp@gmail.com

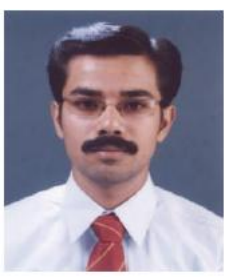

Venkatraman.S is currently working Assistant Professor, Department of ECE, Vel Tech, Avadi, Chennai, India. He has completed his Bachelor Degree in Electronics and Communication (2006), Masters in VLSI Design (2008) and also he completed Masters in Business Administration (2009). He worked for various institutions in electronics and communication department around many states in India. He keeps many international publications on to his 
credit. His research areas are VLSI Physical Design and Testing, Low Power, Nano materials and CAD Algorithms. Email:venkivl18@gmail.com.

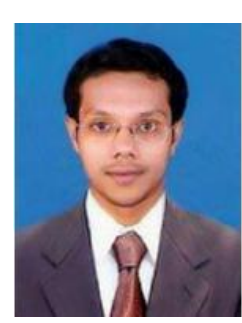

Hameem Shanavas.I is the Doctoral Research Scholar of Anna University, Coimbatore, India. He is currently working Assistant Professor, Department of ECE, M.V.J. College of Engineering, Bangalore, India. He has completed his Bachelor Degree in Electronics and Communication (2006), Masters in VLSI Design (2008) and also he completed Masters in Business Administration (2009). He worked for various institutions in electronics and communication department around many states in India .He had more than 30 publications in international level. $\mathrm{He}$ is in editorial committee of many International Journals like IJESET, WASET and reviewer for many Journals like IEEE Transactions, Science Direct, VLSICS, SIPICS, and IJANS etc. He is the member of Professional bodies like ISECE, IACSIT, and IAEng. His research areas are VLSI Physical Design and Testing. Email:hameemshan@gmail.com. 